\title{
Autoimmune Thyroid, Celiac and Addison's Diseases Related to HLA-DQ Types in Young Patients with Type 1 Diabetes in Belgium
}

\author{
Anissa Messaaoui ${ }^{1}$, Sylvie Tenoutasse ${ }^{1}$, Bart Van der Auwera ${ }^{2},{\text { Christian } \text { Mélot }^{3} \text {, Harry Dorchy }}^{1}$ \\ ${ }^{1}$ Diabetology Clinic, University Children’s Hospital Queen Fabiola, Université Libre de Bruxelles, Brussels, Belgium \\ ${ }^{2}$ Belgian Diabetes Registry, Brussels, Belgium \\ ${ }^{3}$ Statistics Department, Erasme Academic Hospital, Université Libre de Bruxelles, Brussels, Belgium \\ Email: anissa.messaaoui@huderf.be
}

Received August 24, 2012; revised September 25, 2012; accepted October 27, 2012

\begin{abstract}
Objective: To define the prevalence of auto antibodies (Ab) to different organs in young patients with type 1 diabetes (type 1DM). Methods: Ab to thyroid, celiac and adrenal disease was analyzed in 831 type 1 DM patients. Results: Hundred twenty-three (14.8\%) had positive thyroid Ab. The risk of developing thyroid Ab was increased in girls (HR 2.3; 95\% CI 1.6 - 3.2, $\mathrm{p}<0.0001)$. Thirty-three (3.9\%) patients had positive endomysium Ab (3.1\% in girls and 3.5\% in boys, $\mathrm{p}=\mathrm{NS}$ ). Adrenal Ab was detected in 5 patients. The DQA1*0301-DQB1*0301 haplotype was more prevalent in patients with thyroid Ab ( $\mathrm{p}=0.0281)$; DQA1*301-DQB1*302 and DQA1*501-DQB1*201 in patients with endomysium $\mathrm{Ab}(\mathrm{p}=0.0251$ and $\mathrm{p}<0.0001)$. All patients with adrenal Ab were DQA1*301-DQB1*302 positive. Conclusions: Type 1D patients should be screened annually for thyroid autoimmunity and celiac disease. The DQA1*0301-DQB1*0301 haplotype seems to confer susceptibility to thyroid autoimmunity, DQA1*301-DQB1*302 and DQA1*501-DQB1*201 to celiac disease and DQA1*301-DQB1*302 to adrenal autoimmunity.
\end{abstract}

Keywords: Type 1 diabetes; Autoimmunity; Thyroid Disease; Celiac Disease; HLA-DQ Type

\section{Introduction}

Type 1 diabetes (type $1 \mathrm{DM}$ ) is an autoimmune endocrine disease. It is characterized by $\mathrm{T}$ cell infiltration and production of autoantibodies directed to the pancreatic islets resulting in their dysfunction and destruction [1]. This process occurs in genetically susceptible persons, is probably triggered by one or more environmental agents and progresses over many months. Insulin deficiency following destruction of the insulin-producing pancreatic beta cells causes hyperglycemia and diabetic ketoacidosis [1].

Immune dysregulation and attack can affect other organs [2]. In patients with type $1 \mathrm{DM}$, other autoimmune diseases like autoimmune thyroid (Hashimoto's thyroiditis or Graves' disease), celiac and Addison's disease can be present. They are associated with organ-specific autoantibodies: thyroid peroxidase and thyroglobulin with autoimmune thyroid disease, endomysial autoantibodies with celiac disease and 21-hydroxylase autoantibodies with Addison's disease [3].

Their similar pathogenesis and their tendency to occur together suggest that the etiology of type $1 \mathrm{DM}$ and other autoimmune diseases may involve common genetic factors. Different genes have been analysed like the major histocompatibility complex (MHC), the MHC I-related gene A, the PTPN22 gene, the cytotoxic T lymphocyte-associated antigen- 4 gene [4] $\cdots$ Nevertheless the exact etiology of the immune response in these autoimmune diseases is still unclear.

Much of the familial risk for developing type $1 \mathrm{DM}$ and associated autoimmune diseases is provided by human leukocyte antigen (HLA) genotypes. The MHC genes are the most important type $1 \mathrm{DM}$ susceptibility genes and are located on chromosome 6p21 [5]. Different MHC genes were found to confer either susceptibility or protection for type 1 DM. HLA-DQ genes constitute more or less $50 \%$ of the genetic risk of type 1 diabetes. The most specific haplotype for type 1 diabetes is the DQA1*0301-DQB1*0302/DQA1*0501-DQB1*0201 [6]. The MHC has also been extensively studied in autoimmune thyroid, celiac and Addison's diseases.

The aim of this study was to define the prevalence of autoantibodies to different organs in a large cohort of young patients with type $1 \mathrm{DM}$ and to link their occurrence with clinical data and the HLA-DQ type. 


\section{Subjects and Methods}

\subsection{Patients}

A total of 850 patients with type $1 \mathrm{DM}$ were recruited by the Diabetology Clinic of the University Children's Hospital Queen Fabiola. Data were available for analysis from 831 patients (367 females) aged [median (interquartile range)] 18.0 (13.1 - 25.6) years followed for 8.7 (4.1 - 15.1) years. Median age of type 1 DM diagnosis was 9.0 (5.1 - 12.4) years. The medical ethics committee of the hospital approved the study.

\subsection{Methods}

Auto-antibodies (Ab) against thyroid peroxidase (TPO), thyroglobulin (TG), endomysium (EM Ab) and adrenal gland were determined each year since the onset of diabetes. Antibodies anti-TPO, anti-TG were measured on a chemi-luminescence analyzer Advia Centaur (Siemens Healthcare Diagnostics, Deerfield IL). Anti-endomysium and anti-adrenal antibodies were detected by an indirect immuno-fluorescence assay using, respectively, sections of primate distal oesophagus (A. Menarini Diagnostics, Zaventhem Belgium) and primate adrenal tissue slides (INOVA Diagnostics, San Diego) following manufacturer's recommendations. Conjugate anti-human IgG/A/ M FITC was provided by Bio-Rad. Slides were observed on a Leica DM2000 fluorescence microscope (magnification $x 500,50 x / 0.90$ lens). In patients with positive TPO and/or TG Ab, thyroid function tests (TSH and free T4 levels) were measured. L-thyroxine treatment was started if TSH was higher than $4.5 \mathrm{mU} / \mathrm{l}$. In patients with positive EM Ab, biopsy of the small intestine by upper endoscopy was performed. In 694 patients, HLA-DQ genotype was determined by the Belgian Diabetes Registry using polymerase chain reaction and oligotyping with allele specific probes as described previously [6]. No sample for HLA-DQ typing was available for the remaining 137 patients.

\subsection{Statistical Analysis}

None of the variables analyzed were normally distributed. Data are presented as median (interquartile range). Wilcoxon's Rank Sum test was used for comparisons. Differences of frequencies for categorical variables were tested by $x^{2}$ test. Cumulative risk of developing antibodies to thyroid was calculated. The log rank test was used for comparison of gender and hazard ratio (HR) with 95\% confidence interval (CI) was calculated. A p-value $<0.05$ was considered to be significant.

\section{Results (Table 1)}

\subsection{Autoimmune Thyroid Disease}

Hundred twenty-three (14.8\%) patients had positive an- tithyroid peroxydase and/or thyroglobulin antibodies. The risk of developing thyroid antibodies was increased in female (HR 2.3; 95\% CI 1.6 - 3.2; p < 0.0001, Figure 1). Diabetes duration was $5.2(1.7$ - 10.0) years before autoantibodies to thyroid appearance. Patients with thyroid antibodies were older, had a longer duration of diabetes compared with patients without antibodies. There was no difference in the age at diabetes diagnosis between patients with and without antibodies. Twelve patients (11 females) required treatment.

\subsection{Celiac Disease}

Thirty-three (3.9\%) patients had positive EM Ab (3.1\% in girls and 3.5\% in boys; $\mathrm{p}=\mathrm{NS}$ ). Diabetes duration was 7.4 (2.8 - 12.9) years before celiac antibodies appearance. Patients with celiac antibodies were younger, younger at diabetes diagnosis and had a longer duration of diabetes compared with patients without celiac antibodies. Thirtyone biopsies were performed. Twenty-nine (3.5\%) patients required gluten-free diet. The histologic features range from a mild alteration characterized only by increased intraepithelial lymphocytes, to a flat mucosa with total mucosal atrophy, complete loss of villi, enhanced epithelial apoptosis and crypt hyperplasia.

\subsection{Addison's Disease}

Adrenal autoantibodies were detected in five patients (3 females) after a 7.5 (2.0 - 15.6) years diabetes duration. None had Addison's disease.

The presence of thyroid, celiac or adrenal antibodies were independent $(p=N S)$.

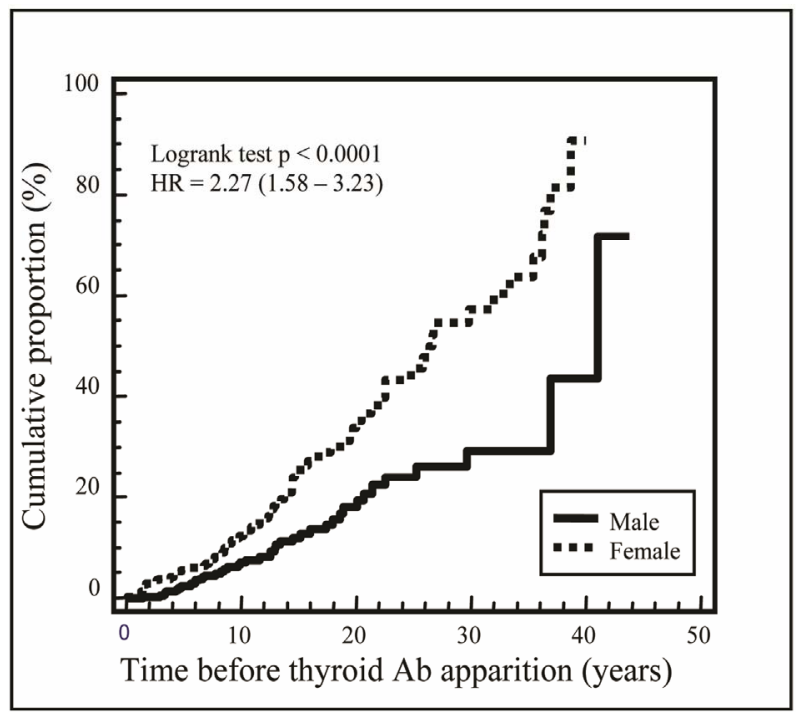

Figure 1. Cumulative incidence for autoantibodies to thyroid apparition from diabetes diagnosis: the risk of developing thyroid autoimmunity was increased in girls with type 1 diabetes. 
Table 1. Clinical data in young patients with type 1 diabetes with and without antibodies to thyroid and celiac disease.

\begin{tabular}{cccc}
\hline Thyroid disease & Positive thyroid Ab & Negative thyroid Ab & p \\
\hline Number (\%) & $123(14.8)$ & $708(85.2)$ & 0.0004 \\
Age (years) & $21.0(14.5-30.8)$ & $17.5(12.8-24.9)$ & 0.0003 \\
Diabetes duration (years) & $11.6(6.0-18.9)$ & $8.2(3.7-14.7)$ & NS \\
Age at diabetes diagnosis (years) & $8.3(5.2-13.3)$ & $9.1(5.0-12.2)$ & Negative celiac Ab \\
\hline Celiac disease & Positive celiac Ab & $690(83.0)$ & - \\
Number (\%) & $141(17.0)$ & $18.6(13.5-26.7)$ & 0.0001 \\
Age (years) & $15.8(11.4-21.4)$ & $8.8(4.3-15.4)$ & 0.0134 \\
Diabetes duration (years) & $7.4(2.8-12.9)$ & $9.3(5.3-12.6)$ & 0.0116 \\
\hline
\end{tabular}

\subsection{HLA-DQ typing}

Seventy-one percent of the 694 patients with HLA DQ typing were HLA DQA1*0501-DQB1*0201 and/or DQA1*0301-DQB1*0302 positive. The DQA1*0301DQB1*0301 haplotype was significantly for celiac disease especially for young patients [7]. Anti-thyroid Ab positivity indicates the necessity for thyroid function testing and EM Ab the necessity for biopsy. Fortunately, the additional diagnosis of such diseases has minimal impact on quality of life in children with Type 1 DM [8]. In absence of Addison's disease signs, systematic annual screening of adrenal $\mathrm{Ab}$ in patients with Type $1 \mathrm{DM}$ should not be recommended. The DQA1*0301-DQB$1 * 0301$ haplotype seems to confer susceptibility to thyroid autoimmunity, haplotypes DQA1*0301-DQB1*0302 and DQA $1 * 0501-\mathrm{DQB} 1 * 0201$ to celiac disease and DQA1*0301-DQB1*0302 to adrenal autoimmunity.

\section{Discussion}

Our results confirm in a large group of young patients with type $1 \mathrm{DM}$ that they should be screened annually for thyroid autoimmunity [9] especially for females [10] and for celiac disease especially for young patients [7].

Anti-thyroid Ab positivity indicates the necessity for thyroid function testing and EM Ab the necessity for biopsy. Fortunately, the additional diagnosis of such diseases has minimal impact on quality of life in children with Type 1 DM [8]. In absence of Addison's disease signs, systematic annual screening of adrenal Ab in patients with Type $1 \mathrm{DM}$ should not be recommended. The DQA1*0301-DQB1*0301 haplotype seems to confer susceptibility to thyroid autoimmunity, haplotypes DQA1*0301-DQB1*0302 and DQA1*0501-DQB1*0201 to celiac disease and DQA1*0301-DQB1*0302 to adrenal autoimmunity.

\section{Acknowledgements}

We thank the Belgian Diabetes Registry, especially Ilse Weets, for their contributions.

\section{REFERENCES}

[1] J. A. Todd, “Etiology of Type 1 Diabetes," Immunity, Vol. 32, No. 4, 2010, pp. 457-467. doi:10.1016/j.immuni.2010.04.001

[2] H. Dorchy, B. Lemiere, D. Toussaint and P. Gausset, "Islet-Cell Antibodies and Organ-Specific Antibodies in Diabetic Children and Adolescents," Nouvelle Presse Médicale, Vol. 10, No. 34, 1981, pp. 2795-2798.

[3] T. M. Triolo, T. K. Armstrong, K. McFann, L. Yu, M. J. Rewers, G. J. Klingensmith, G. S. Eisenbarth and J. M. Barker, "Additional Autoimmune Disease Found in 33\% of Patients at Type 1 Diabetes Onset,” Diabetes Care, Vol. 34, No. 5, 2011, pp. 1211-1213. doi:10.2337/dc10-1756

[4] J. M. Barker, "Clinical Review: Type 1 Diabetes-Associated Autoimmunity: Natural History, Genetic Associations and Screening,” The Journal of Clinical Endocrinology \& Metabolism, Vol. 91, No. 4, 2006, pp. 1210-1217. doi:10.1210/jc.2005-1679

[5] A. G. Ziegler and G. T. Nepom, "Prediction and Pathogenesis in Type 1 Diabetes,” Immunity, Vol. 32, No. 4, 2010, pp. 468-478. doi:10.1016/j.immuni.2010.03.018

[6] H. Heimberg, Z. P. Nagy, G. Somers, I. De Leeuw and F. C. Schuit, "Complementation of HLA DQA and -DQB Genes Confers Susceptibility and Protection to Insulin-Dependent Diabetes Mellitus,” Human Immunology, Vol. 33, No. 1, 1992, pp. 10-11. doi:10.1016/0198-8859(92)90046-P

[7] J. M. Barker, J. Yu, L. Yu, J. Wang, D. Miao, F. Bao, E. Hoffenberg, J. C. Nelson , P. A. Gottlieb, M. Rewers and G. S. Eisenbarth, “Autoantibody 'Subspecificity' in Type 1 Diabetes,” Diabetes Care, Vol. 28, No. 4, 2005, pp. 850-855. doi:10.2337/diacare.28.4.850 
[8] S. Sud, M. Marcon, E. Assor, D. Daneman and F. H. Mahmud, "Quality of Life in Children with Diabetes and Celiac Disease: Minimal Impact of the 'Double Diagnosis',” Pediatric Diabetes, Vol. 13, No. 2, 2012, pp. 163169. doi:10.1111/j.1399-5448.2011.00785.x

[9] E. Piatkowska and M. Szalecki, "Autoimmune Thyroiditis in Children and Adolescents with Type 1 Diabtetes,"
Pediatric Endocrinology, Diabetes, and Metabolism, Vol. 17, No. 4, 2011, pp. 173-177.

[10] O. Kordonouri, A. Klinghammer, E. B. Lang, A. Gruters-Kieslich, M. Grabert and R. W. Holl, "Thyroid Autoimmunity in Children and Adolescents with Type 1 Diabetes," Diabetes Care, Vol. 25, No. 8, 2002, pp. 13461350. doi:10.2337/diacare.25.8.1346

\section{Abbreviations}

Ab: antibodies;

EM: endomysium;

HLA: human leukocyte antigen;

MHC: major histocompatibility complex;

Type 1 DM: type 1 diabetes;

TG: thyroglobulin;

TPO: thyroid peroxidase. 